The pulping tests established definitely the difficulty of dealing with wood containing appreciable amounts of heartwood, and an increased temperature-gradient and penetration-period were required in such cases. The resin-contents actually obtained were the very low figure of 0.35 per cent for pulps from sapwood, and 3.84 per cent from heartwood pulps. No pitch trouble was experienced with the former, but the indications are that the latter would give trouble from this source.

J. G.

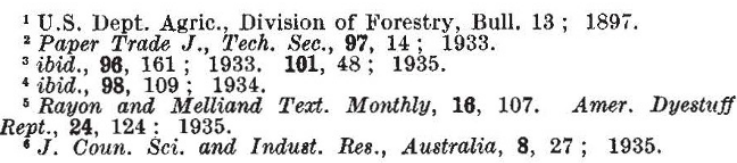

\title{
Recent Research on Cancer
}

$\mathrm{T}$ HE twelfth annual report of the British Empire Cancer Campaign gives summaries of the past year's progress in most of the cancer research centres of the British Empire. During the year, more than $£ 32,000$ has been distributed by the Campaign in encouraging and supporting research along a very broad front, extending from pure physics, such as methods of inducing radioactivity in normally inactive elements, to the study of the origin and development of tumours in the human body. With the hope of improving and extending clinical research, a new clinical research committee has been formed with the cooperation of all the London teaching hospitals. Such an organisation can help considerably in comparing results and preventing overlapping and duplication of research.

The problem of the cause of cancer has bəen attacked mainly by means of experimental carcinogenesis, in many different institutes. Many vears ago, Fibiger found that gastric cancer occurred in rats fed on cockroaches infected with Gongylonema neoplasticum, when the rats become hosts for the parasites. Prof. R. D. Passey has now found that infected rats do not develop cancer if they are fed on a full and healthy diet in place of the white bread diet used in Fibiger's experiments. This indicates that diet may influence the occurrence of malignant disease. In the Cancer Hospital Research Institute a new, very active carcinogenic compound, methyl cholanthrene, has been produced from deoxycholic acid (a normal constituent of bile), and has been synthesised. In structure, methyl cholanthrene is allied to sterols and sex hormones and to other carcinogenic agents, and it is conceivable that a similar active carcinogenic compound might arise adventitiously in the body by some 'error' of metabolism. This suggests a possible link between industrial cancer, such as that of gas workers and mule spinners, and natural or spontaneous cancer.

Sarcomata have been induced in fowls by synthetic carcinogenic agents and by tar in many different laboratories, and attempts have been made to obtain a filterable virus from the neoplasms so obtained. Prof. J. McIntosh has described three tar-induced sarcomata capable of propagation by cell-free filtrates. On the other hand, careful work carried out in Sheffield, Glasgow and Edinburgh, with fowl tumours induced by $1: 2: 5: 6$-dibonzanthracene, has not given any evidence of a filterable virus in these tumours. Prof. E. Mellanby has found that the virus of the Rous chicken sarcoma, which can be propagated by cell-free filtrates, is present, not only in the normal organs of infected birds, but also in dibenzanthracene tumours of birds bearing the filterable tumour. If a fowl bearing a tumour induced by $1: 2: 5: 6$-dibenzanthracene is infected with Rous chicken sarcoma, the virus of the latter passes into the dibenzanthracene induced tumour, so that an extract of the dibenzanthracene tumour injected into other fowls produces tumours resembling the Rous tumour and not the dibenzanthracene tumour.

In the study of tumour viruses, the high-speed centrifuge is being developed into a useful tool. The air-driven and air-floating centrifuge is relatively simple and inexpensive, and as it rotates at 40,000-60,000 r.p.m. it makes it possible to subject liquids to forces up to 70,000 times that of gravity. By means of this centrifuge it is possible to concentrate the active agent of fowl sarcomata. The concentrates contain elementary bodies, possibly virus particles, which can be examined under the microscope.

Work on the nature of the changes occurring in isolated cancer tissue continues to give interesting results, but the problem of the anomalous high rate of lactic acid formation in the presence of oxygen remains unsolved. Dinitro-phenols which cause increases in temperature and metabolism in man and animals also increase the respiration and lactic acid production of isolated tumour tissuethe mechanism of the biological effect of nitrophenols is still obscure. On the other hand, 
naturally occurring coloured oxygen carriers such as pyocyanine and lactoflavine increase the oxygen consumption and decrease the lactic acid formation of tissues. Dr. F. Dickens has found that other dyes which are photo-sensitisers, such as phenosafranine, cause increased aerobic lactic acid production. It is remarkable that some irritants such as mustard gas (dichloro diethyl sulphide) and cantharidine prevent or delay the carcinogenic action of tar or similar agents. Dr. I. Berenblum and Dr. L. P. Kendal found that these anti-carcinogenic agents are able to inhibit glycolysis to a greater extent than respiration. It seems to the present writer that this effect may be allied to the effect of iodoacetic acid inhibiting the lactic acid production of muscle.

In the recently formed Cancer Department of St. Bartholomew's Hospital, a million volt X-ray plant is being installed. This should give a more powerful source of short-wave radiation than any radium bomb or X-ray plant at present in use. The results from this plant will be of extreme interest. The present report gives data referring to the radiological treatment of patients in many hospitals, and the pooling of results in this way must be of value in assessing the value of radiological methods in clinical work.

Radiations from radium and X-ray tubes have been used in many experiments on chick embryo and on tadpole tissues. Gamma-rays are found to have most effect on cells that are about to divide ; once cell division has commenced, the process completes itself in a normal manner even in the presence of gamma-rays. The first effect of gamma-radiation is therefore apparently to reduce mitotic activity; but this is followed in turn by periods of apparent recovery, degeneration, abnormal mitosis and final recovery. Further examination of the effect of radium on tissue cultures of chick embryo tissue seems to indicate that the lethal effect depends on the actual amount of radiation rather than the time over which the radiation is spread.

Several specimens of human carcinoma removed in operation have been grown in vitro. An interesting phenomenon demonstrated by Dr. T. Lumsden with tissue culture methods is the action of antibodies capable of killing cancer cells in vitro. The effect of the anti-cancer sera has now been photographed with a cinema camera, and the death of tissue culture cells in the presence of the serum is strikingly shown in the film. It would be of interest to see the effect of the rat anti-cancer serum on tissue cultures of human carcinomata.

It was shown some time ago that snake venom had a toxic effect on malignant growths. The toxic effect of cobra venom on tumours is not prevented by anti-venom serum, and it may be possible to check the toxic effect of the venom on the host without inhibiting the effect on the cancerous growth. This is possibly a hopeful method of attacking the problem of curing cancer. With the active research that is being prosecuted along so many divergent lines in many laboratories, it is possible to say that advances are being continually made, but by which way the goal will be attained it is quite impossible to forecast.

\section{Obituary}

\section{Prof. Charles Richet}

A STONE'S throw from the tip of the headland of Giens, in the south of France, there is a cluster of rocky islets. One of the most picturesque of them is pointed at with awe by the local country folk, as the home of a famous old man who carried out mysterious experiments and tried to communicate with the world beyond. It was, indeed, the country home of Prof. Charles Richet, well known in physiology and abnormal psychology, who died on Decem. ber 3 in Paris.

Physiology and medicine owe to Richet the discovery of anaphylaxis, in 1902, which marked a decisive advance in modern medicine, and for which he was awarded the Nobel Prize for Medicine in 1913. This discovery was the result of a series of experiments and researches dating so far back as 1887, a year before the foundation of the Pasteur
Institute, when Richet was the first to carry out an injection of serum into a human being. He was thus one of the founders of serotherapy.

In 1902, Richet was studying with Portier the action of the poison of sea-anemones, when they remarked that dogs which had withstood without any inconvenience an intravenous injection of a minimal dose of an aqueous extract of the tentacles of Actinia, always died after a second but weaker injection administered to them in the same way a few days later. But as this second injection was not strong enough to kill a fresh animal, they came to the conclusion that the first dose of a serum does not always immunise, but on the contrary, renders the organism more sensitive for a certain period and though the effect of the first dose seems to have disappeared. Thus were cleared up many mysterious cases of intoxication and an explanation was forth- 